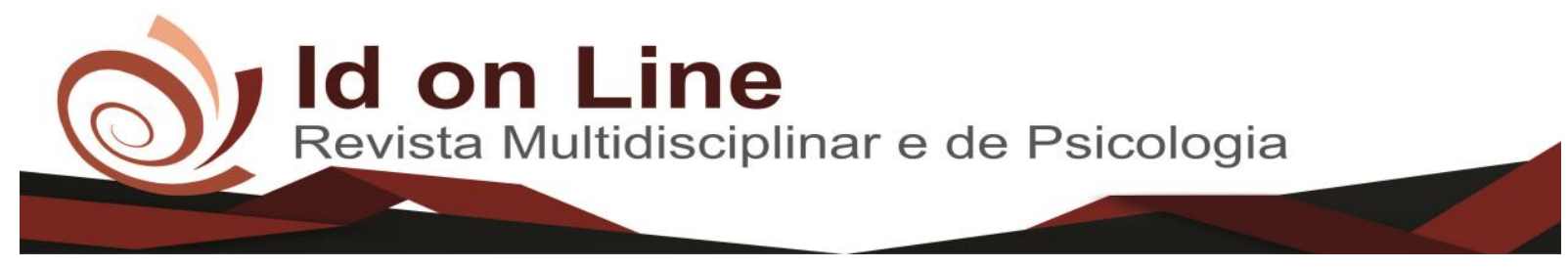

Artigo de Revisão

\title{
Conhecimento dos Cirurgiões-Dentistas da rede pública sobre câncer bucal: Revisão de literatura
}

\author{
Brenda Sousa da Silva ${ }^{1}$; Gefter Thiago Batista Corrêa ${ }^{2}$; Karine Brasileiro de Oliveira ${ }^{3}$, \\ Amanda Macedo Rosa Simões ${ }^{4 ;}$ Lara Correia Pereira ${ }^{5}$
}

Resumo: O câncer bucal é apontado como um problema de saúde pública, considerado potencialmente fatal, com elevada incidência global. Considerando a importância da prevenção e do diagnóstico precoce da neoplasia, a atenção primária à saúde é uma ambiência adequada para o desenvolvimento dessas ações, e a atenção odontológica exerce papel estratégico nesse enfrentamento. O objetivo desse trabalho é averiguar, por meio de uma revisão de literatura, o nível de conhecimento dos cirurgiões-dentistas sobre o diagnóstico do câncer bucal e sua prevenção. O papel do cirurgião-dentista é de extrema importância nos diversos níveis de atenção a saúde, além de ser responsável também pela articulação e criação de práticas e políticas que diminuam a exposição aos fatores de risco e introduzam na população uma consciência quanto à importância da prevenção e do diagnóstico precoce do câncer.

Palavras-chave: Câncer oral. Saúde Pública. Cirurgiões-dentistas. Diagnóstico precoce.

\section{Knowledge of dental surgeons of the public network on oral cancer: Literature review}

\begin{abstract}
Oral cancer is regarded as a potentially fatal public health problem with a high global incidence. Considering the seriousness of the prevention and early diagnosis of neoplasia, primary health care is an adequate environment for the development of these actions, and dental care plays a strategic role in this confrontation. The objective of this study is to investigate, through a literature review, the level of knowledge of dental surgeons on the diagnosis of oral cancer and its prevention. The role of the dental surgeon is of extreme importance at the various levels of prevention, as well as being responsible for articulating and creating practices and policies that reduce exposure to risk factors and introduce in the population an awareness of the importance of prevention and early diagnosis of cancer.
\end{abstract}

Key words: Oral cancer. Public health. Dental surgeons. Early diagnosis.

\section{Introdução}

O câncer é uma doença que necessita de uma maior compreensão quanto às suas causas, demandando estudos que viabilizem tratamentos cada vez mais eficientes e que progressivamente se aproxime da cura.

\footnotetext{
${ }^{1}$ Discente do Curso de Odontologia da Faculdade Independente do Nordeste. Contato: brendasousa011@hotmail.com;

${ }^{2}$ Mestre em Ciências da Saúde com ênfase em patologia bucal. Doutor em Medicina Molecular. Professor da Faculdade Independente do Nordeste - FAINOR:

${ }^{3}$ Cirurgiã- Dentista. Especialista em Paciente com Necessidades Especiais. Especialista em Odontologia Estética;

${ }^{4}$ Discente do Curso de Odontologia da Faculdade Independente do Nordeste;

${ }^{5}$ Mestre em Estomatologia Bucal. Especialista em Prótese Dentária. Professora da Faculdade Independente do Nordeste - FAINOR.
} 
É uma doença que se desenvolve de modo gradual, ocasionando em grande parte a morte dos pacientes acometidos por essa patologia. Quando uma célula normal passa por algumas mutações dando origem a outra célula capaz de se desdobrar de forma desmedida se espalhando por todo o organismo denomina-se câncer ${ }^{1}$.

De acordo com dados do Instituto Nacional de Câncer (INCA) são detectados mais de 12,7 milhões de casos de câncer em todo o mundo, com 7,6 milhões de mortes a cada ano. Caso não sejam tomadas devidas providências, em 2030 estima-se um quadro de 26 milhões de novas ocorrências ${ }^{1}$.

Com a frequência das ocorrências de câncer registradas constantemente em todas as nações e diante das alarmantes progressões mundiais, torna-se imprescindível uma abordagem sucessiva desse tema com o intuito de ampliar o conhecimento da população e consequentemente, minimizar o número de $\operatorname{casos}^{2,3}$.

O Carcinoma de Células Escamosas (CCE), ou carcinoma espinocelular, é uma neoplasia de origem epitelial, que apresenta uma prevalência de mais de $90 \%$ de todas as neoplasias que afetam o trato aero digestivo superior. Clinicamente, o CCE se apresenta como uma lesão ulcerada, com base endurecida, centro necrosado, bordas elevadas e nítidas, por efeito da absorção dos tecidos subjacentes, normalmente assintomático em seu início e de acelerado crescimento ${ }^{2,3}$.

O desenvolvimento do câncer oral é ocasionado pela influência de fatores ambientais e os hábitos de vida dos indivíduos, podendo ser constatado em todas as pessoas, mas com maior incidência nos homens. O perfil clássico dos pacientes acometidos por carcinoma é: sexo masculino, acima de 40 anos de idade, de baixa classe educacional e socioeconômica. De etiologia multifatorial, o tabaco e o álcool intensificam o risco do desenvolvimento da doença conforme a quantidade usada, estando associados à mais de $80 \%$ dos $\operatorname{casos}^{4}$.

O diagnóstico precoce é uma ferramenta importante para prevenção e melhoria do prognóstico do paciente. Nos estágios iniciais, o CCE apresenta índices de cura de aproximadamente $90 \%$ dos $\operatorname{casos}^{4}$. Nos casos de lesões em cavidade oral, há um foco maior no profissional cirurgião-dentista, em estar habilitado à desenvolver um exame clínico completo e possíveis exames complementares, afim de fechar um diagnóstico preciso ${ }^{5}$.

A interferência do cirurgião-dentista abrange variados níveis de prevenção, além deste ser encarregado também pela criação e articulação de práticas e políticas que diminuam a 
exposição dos pacientes aos fatores de risco e conscientizem a população o quanto são importantes a prevenção e o diagnóstico precoce ${ }^{6}$.

O autoexame bucal é um dos métodos mais significativos para se adquirir o diagnóstico do câncer oral em fase inicial, proporcionando significativa melhora para o prognóstico do paciente. Tal procedimento deveria ser minuciosamente explicado nas atividades de educação comunitária e nas consultas iniciais, com linguagem acessível e fácil à população. Todavia, a maior parte da população desconhece este fato ${ }^{7}$.

Desse modo, o atendimento à atenção básica da família trouxe um grande avanço incorporando o cirurgião-dentista na promoção de ações de saúde bucal. No Brasil, a inserção desse profissional no serviço público foi reforçada desde o ano 2000, com as chamadas Equipes de Saúde Bucal, garantindo prevenção e tratamento bucal de forma gratuita à população ${ }^{8}$.

O empenho no trabalho de divulgação desse método, assim como para o diagnóstico e tratamento eficazes do câncer, na atenção básica não se limita ao cuidado do profissional de saúde oral. Trata-se de um desafio de saúde pública, fazendo com que todos profissionais envolvidos no âmbito da saúde primária se abarquem por essa problemática ${ }^{9}$.

Assim, o objetivo desse trabalho é averiguar, por meio de uma revisão de literatura, o nível de conhecimento dos cirurgiões-dentistas da rede pública sobre o diagnóstico do câncer bucal e sua prevenção.

\section{Metodologia}

O presente estudo corresponde a uma revisão de literatura baseada na análise de artigos científicos publicados de 2007 a 2018. A busca pela literatura foi norteada por termos relacionados ao câncer de boca, fatores de risco, diagnóstico e importância do cirurgião-dentista de forma combinada, nos idiomas espanhol, inglês e português. Ademais a consulta bibliográfica ainda abrange abstracts, monografias, teses, livros e os sites do Instituto Nacional do Câncer - INCA e da Associação Brasileira de Saúde Coletiva - ABSC. Para a pesquisa foram utilizadas as bases de dados PUBMED, LILACS e BIREME. 
Sendo que a seleção dos artigos teve como critérios de inclusão a correlação com o tema abordado e publicações referentes aos últimos onze anos. Descartando aqueles publicados anterior a esse período.

\section{Revisão de Literatura}

Pode-se definir o câncer como sendo o crescimento descontrolado de células provenientes de outra, a princípio normal, mas que tenha passado por alterações sendo capaz de se dispersar em todo o organismo ${ }^{10}$. Tais células são propensas à formação de tumores malignos na medida em que se propagam de forma desordenada atuando de maneira extremamente agressiva. No campo da Odontologia, o câncer de boca tem se destacado nas últimas décadas, haja vista que sua incidência no Brasil é considerada uma das mais altas no mundo ${ }^{11}$.

Trata-se de uma doença multifatorial, resultante da interação dos fatores de riscos que afetam os processos de controle, proliferação e crescimento celular. Sendo os principais fatores de risco: o álcool, o tabaco, a radiação solar, a dieta, os microorganismos e a deficiência imunológica, destacando os três primeiros fatores. O cigarro, por exemplo, é considerado como o principal agente carcinogênico responsável por atuar sobre as células iniciadas, transformando-as em malignas, denominado agente oncopromotor ${ }^{10,12 .}$.

Os tumores que acometem as regiões anatômicas da boca apresentam diversas manifestações clínicas que podem incluir lesões que persistem por mais de 15 dias; manchas vermelhas ou brancas na língua, gengivas, revestimento da boca; caroços localizados na boca ou no pescoço ${ }^{13}$. E na medida em que essas lesões se tornam mais graves, as atividades de mastigar e engolir ficam cada vez mais árduas, provocando também alterações na fala, enfraquecimento dos dentes e mau hálito ${ }^{14}$.

Conforme evidência a Associação Brasileira de Saúde Coletiva (ABSC) os indicadores epidemiológicos do câncer no mundo demonstram que, o índice de mortalidade se encontra mais frequente nas nações pobres, apesar de que a maioria das ocorrências estejam nos países $\operatorname{ricos}{ }^{15}$. 
De acordo com o INCA estima-se, para o Brasil, biênio 2018-2019, a ocorrência de 600 mil novos casos de câncer, para cada ano, sendo que dentro desses dados encontra-se 11.200 casos novos de câncer da cavidade oral em homens e 3.500 em mulheres ${ }^{16}$.

O reconhecimento precoce das lesões malignas é importante na melhoria do prognóstico, desenvolvendo notáveis probabilidades de cura e expandindo a qualidade de vida dos pacientes acometidos pela doença, já que essa neoplasia interfere diretamente na funcionalidade do indivíduo e reflete nos índices de sobrevida ${ }^{16,17}$.

A prevenção dessa doença se dá pela forma mais eficaz e simples de combate que é, particularmente, pela ênfase na promoção da saúde, aumento do acesso aos serviços de saúde e diagnóstico precoce ${ }^{18}$. Uma vez que o câncer bucal seja detectado previamente, adquire um maior potencial de controle da doença prolongando a sobrevida de pacientes oncológicos ${ }^{19,20}$.

Nessa perspectiva, o diagnóstico precoce é indiscutivelmente um fator determinante na remissão da doença, minimizando o índice de casos e o número de mortes decorrentes do câncer. Além do mais, o diagnóstico tardio induz a tratamentos mais agressivos, fragilizando o indivíduo e acarretando manifestações emocionais que trazem um sentimento de incerteza e, por vezes, leva à depressão, tornando ainda mais difícil a recuperação do paciente ${ }^{16,21}$.

Embora as lesões bucais sejam facilmente observadas, os pacientes com câncer bucal ainda procuram o serviço de saúde tardiamente, momento em que a doença está em fase bem avançada, normalmente por não valorizar os primeiros sintomas, seja por falta de esclarecimento ou até mesmo pela dificuldade no acesso ao serviço especializado e por isso, o retardo do diagnóstico trás, como consequência, tumores maiores e mais destrutivos com tratamentos complexos e índices de mortalidade elevados ${ }^{22}$.

Em virtude destes fatos, é essencial desenvolver campanhas informativas em saúde oral que conscientize a população sobre como suas condições de vida induzem no surgimento do câncer de boca, bem como instruir sobre a importância de detectar a doença em seu estágio inicial, visto que a falta de conhecimento da população é uma das principais causas do retardo do diagnóstico ${ }^{23}$.

No Brasil, é de competência do Sistema Único de Saúde (SUS) elaborar ações no âmbito da saúde pública, oferecendo assistência de modo gratuito que abranja toda a população ${ }^{24}$. Essa interação com a comunidade pode ocorrer a partir das visitas domiciliares dos agentes comunitários, cabe a esses profissionais que atuam na atenção básica construir uma relação com 
os moradores de sua abrangência, notificando os indicadores de saúde, intervindo de maneira preliminar e direcionando os quadros agravados para o devido atendimento.

Diante disso, o governo federal criou o programa intitulado Brasil Sorridente, com o intuito de promover ações gratuitas de prevenção e recuperação da saúde bucal da população brasileira. O projeto conta com 23.175 equipes de saúde bucal, que atendem nas unidades básicas de saúde de todo o país, número esse quase quatro vezes maior que o total antes da concepção, 6.170 equipes. Em onze anos do programa, o total de dentistas atuando no SUS cresceu cerca de 50\%. e, atualmente, o SUS emprega 30\% dos dentistas do país, contando com uma equipe de 64,8 mil profissionais ${ }^{25}$.

Nesse caso, o cirurgião-dentista é o profissional da área da odontologia capacitado para prevenir, diagnosticar e tratar problemas bucais, sua função vai muito além da estética e dos procedimentos clínicos relativos a boca, englobando a vigilância epidemiológica, sendo portanto, responsável por práticas educativas de esclarecimento quanto aos cuidados à saúde bucal, assegurando a população um conhecimento prévio sobre anomalias como o câncer de boca, bem como os fatores decorrentes dessa doença ${ }^{26}$.

À vista disso, a intervenção do cirurgião-dentista na detecção precoce do câncer oral é seguramente fundamental, de modo que durante as consultas odontológicas este profissional deve ser capaz de identificar lesões iniciais muitas vezes despercebidas pelos pacientes por serem assintomáticas em sua fase inicial. Além do mais, o cirurgião-dentista deve estar apto para orientar seus pacientes quanto aos fatores de risco e as formas de prevenção, incentivando a prática do autoexame que consiste na busca de alterações orais incomuns com o auxílio de um espelho, procedimento esse que melhora consideravelmente o prognóstico do paciente ${ }^{27,28}$.

A partir da detecção da lesão busca-se o tratamento mais adequado, neste caso a remoção do tumor através da intervenção cirúrgica pode ser suficiente quando este é detectado no seu início, direcionando para um prognóstico favorável e dispensando tratamentos severos como a radioterapia e a quimioterapia que são terapias invasivas e provocadoras de diversos efeitos colaterais $^{29,30,20}$.

É válido reafirmar que proporcionar informação à população sobre como é importante promover a saúde bucal, enfatizando a prevenção e a detecção precoce das lesões, seja por meio de ações e programas educativos oferecidos pelo SUS, ou por intermédio do cirurgião-dentista é, de fato, a alternativa mais favorável na redução dos altos índices de incidência do câncer ${ }^{31}$. 


\section{Conclusões}

O cirurgião-dentista exerce um dever primário na prevenção do câncer bucal, ser atento para as lesões possivelmente malignas, com relação ao diagnóstico precoce, gerando um prognóstico benéfico para os pacientes com risco de câncer bucal, sendo um diferencial na promoção de saúde.

É necessário que os cirurgiões-dentistas das unidades básicas de saúde estejam sempre presentes em cursos de educação continuada, que são imprescindíveis para aprimoramento. E com isso, ocorreria maior planejamento em políticas públicas saudáveis que assegurem a adesão de estratégias de redução da morbimortalidade da doença.

\section{Referências}

1. Instituto Nacional do Câncer (INCA). Declaração Mundial contra o Câncer. Rio de Janeiro.

2. Silva SR; Juliano Y, Novo NF; Weinfeld I. Estudo comparativo do conhecimento sobre câncer bucal entre acadêmicos de odontologia. Einstein. 2016;14(3):338-45.

3. Corrêa GTB, Bandeira GA, Cavalcanti BG, et al. Association of 308 TNF-a promoter polymorphism with clinical aggressiveness in patients with head and neck squamous cell carcinoma. Oral Oncology. 2011; 47:888-894.

4. Torres-Pereira CC, Angelim-Dias A,et al. Abordagem do câncer da boca: uma estratégia para os níveis primário e secundário de atenção em saúde. Cad. Saúde Pública. 2012; 28:30-39.

5. Andrade SN, Muniz LV, Soares JMA, Chaves ALF, Ribeiro RIMA. Câncer de boca: avaliação do conhecimento e conduta dos dentistas na atenção primária à saúde. Rev. Bras. Odontol. 2014;71(1): 42-7.

6. Souza JGS, Sá AB, Popof DAV. Comportamentos e conhecimentos de cirurgiões-dentistas da atenção primária à saúde quanto ao câncer bucal. Cad. Saúde Colet. 2016;24 (2): 170-177.

7. Reis WG, Scherer MDA, Carcereri DL. O trabalho do Cirurgião-Dentista na Atenção Primária à Saúde: entre o prescrito e o real. Rio de Janeiro. 2015; 39(104): 56-64.

8. Cimardi ACBS, Fernandes APS. Câncer bucal - a prática e a realidade clínica dos cirurgiões dentistas de Santa Catarina. RFO. Saúde Debate. 2009; 14(2):99-104.

9. Correa LM, Silva LM. O uso da citologia esfoliativa no diagnóstico precoce de alterações celulares induzidas pelo uso do fumo. Revista Científica Eletrônica 2018; 1(1):2-14.

10. Maluf FC, et al. Vencer o câncer. São Paulo: Dentrix, 2014. 512 p. 
11. Santos RCS, Dias RS, Giordani AJ, Segreto RA, Segreto HRC. Mucosite em pacientes portadores de câncer de cabeça e pescoço submetidos à radioquimioterapia. Revista da Escola de Enfermagem da USP 2011; 45(6):1338-1344.

12. Oliveira JMB, Pinto LO, Lima NGM, Almeida GCM. Câncer de Boca: Avaliação do Conhecimento de Acadêmicos de Odontologia e Enfermagem quanto aos Fatores de Risco e Procedimentos de Diagnóstico. Revista Brasileira de Cancerologia 2013; 59(2):211-218.

13. Rodrigues AB, Oliveira PP. Casos clínicos em Oncologia. Iátria, 2013. Machado BCP, Gonçalves LM, et al. Avaliação da qualidade de vida dos pacientes portadores de câncer de cabeça e pescoço no Estado do Maranhão. Revista Brasileira de Pesquisa em Saúde. 2009; 11(4): 62-68.

14. Oliveira JMB, Pinto LO, Lima NGM, Almeida GCM. Câncer de Boca: Avaliação do Conhecimento de Acadêmicos de Odontologia e Enfermagem quanto aos Fatores de Risco e Procedimentos de Diagnóstico. Revista Brasileira de Cancerologia 2013; 59(2):211-218.

15. Associação Brasileira de Saúde Coletiva (ABSC). Controle do câncer: o desafio é desigual.

16. Instituto Nacional do Câncer (INCA). Estimativa | 2018 - Incidência de câncer no Brasil. Rio de Janeiro.

17. Amar A, Rapoport A, et al. Prognostic value of regional metastasis in squamous cell carcinoma of the tongue and floor of mouth. Revista Brasileira de Otorrinolaringologia. 2013; 79(6): 734-737.

18. Costa Jr S, Serra CG. Diagnóstico e Continuidade do Cuidado do Câncer Bucal em pacientes acompanhados pelas Equipes de Saúde Bucal do Programa de Saúde da Família: a experiência do município de Resende, no Estado do Rio de Janeiro. Rev. Cadernos UniFOA. 2011; 6(15): 73-90.

19. Jham BC, Freire ARS. Complicações bucais da radioterapia em cabeça e pescoço. Revista Brasileira de Otorrinolaringologia. 2006; 72(5): 704-708.

20. Avila MF, Jara MD, Rondanelli BM. Carcinoma espinocelular de lengua: estudio de sobrevida a 5 anos. Revista Clínica de Periodoncia, Implantología y Rehabilitación Oral. 2016; 9(1): 74-78.

21. Torres SVS, Sbegue A, Costa SCB. A importância do diagnóstico precoce de câncer bucal em idosos. Revista da Sociedade Brasileira de Clínica Médica. 2016; 14(1): 57-62.

22. Zanetti F, Azevedo MLC, et al. Conhecimento e fatores de risco do câncer de boca em um programa de prevenção para motoristas de caminhão . Revista Odontologia Clínico-Científica. 2011; 10(3): 233-241.

23. França DCC, Pinto MMO, et al. Programa de diagnóstico e prevenção de câncer de boca: Uma estratégia simples e eficaz. Revista Odontológica do Brasil-Central. 2010; 19(49): 159-1661.

24. Andrade JOM, Santos CAST, Oliveira MC. Fatores associados ao câncer de boca: um estudo de caso-controle em uma população do Nordeste do Brasil. Revista Brasileira de Epidemiologia. 2015; 18(4): 894-905. 
25. Souza LRB, Ferraz KD, Pereira NS, Martins MV. Conhecimento acerca do Câncer Bucal e Atitudes frente à sua Etiologia e Prevenção em um Grupo de Horticultores de Teresina (PI). Revista Brasileira de Cancerologia. 2012; 58(1): 31-39.

26. Ministério da Saúde. Brasil Sorridente ultrapassa a marca de 1.000 centros odontológicos.

27. Lemos Junior CA, Alves FA, et al. Câncer de boca baseado em evidências científicas. Rev Assoc Paul Cir Dent. 2013; 67(3): 178-186.

28. Thomaz EBAF, Cutrim MCFN, Lopes FF. A importância da educação como estratégia para prevenção e diagnóstico precoce do câncer oral. Acta Oncológica Brasileira / Fundação Antonio Prudente, 2001.

29. Araújo SSC, Padilha DMP, Baldisserotto J. Saúde Bucal e Qualidade de Vida em Pacientes com Câncer de Saúde Bucal e Qualidade de Vida em Pacientes com Câncer de Cabeça e Pescoço Cabeça e Pescoço. Revista da Faculdade de Odontologia de Porto Alegre. 2007; 48(1/3): 73-76.

30. Bruna FBL, Ítala VD, Paola BB. Pacientes com câncer de cavidade bucal submetidos à cirurgia: representações sociais acerca do adoecimento e tratamento. Revista da Sociedade Brasileira de Psicologia Hospitalar. 2013; 16(1).

31. Gomes FC; Marcondes MS, Balducci I, Anbinder AL. Avaliação do conhecimento sobre o câncer de boca entre participantes de campanha para prevenção e diagnóstico precoce da doença em Taubaté - SP. Revista de Odontologia da UNESP. 2006; 35(4): 327-333.

\section{Como citar este artigo (Formato ABNT):}

SILVA, Brenda Sousa da; CORRÊA, Gefter Thiago Batista; OLIVEIRA, Karine Brasileiro de; SIMÕES, Amanda Macedo Rosa; PEREIRA, Lara Correia. Conhecimento dos Cirurgiões-Dentistas da rede pública sobre câncer bucal: Revisão de literatura. Id on Line Rev.Mult. Psic., 2018, vol.12, n.42, p.10181026. ISSN: 1981-1179.

Recebido: 24/10/2018;

Aceito: 29/10/2018 